Fritzsche, Paul; Niemöller, Sven; Laqua, Daniel; Husar, Peter:

ECG-multichannel frontend for quick stimulus response based on FPGA with implemented real-time, online QRS detection algorithm

Zuerst erschienen in: $\quad$ Biomedical Engineering = Biomedizinische Technik. - Berlin [u.a.] : de Gruyter. - 57 (2012), Suppl. 1, Track-F, p. 619-622.

Erstveröffentlichung: 2012-08-30

ISSN (online): $\quad$ 1862-278X

ISSN (print): $\quad$ 0013-5585

DOI: $\quad 10.1515 /$ bmt-2012-4156

[Zuletzt gesehen: 2019-08-12]

„Im Rahmen der hochschulweiten Open-Access-Strategie für die Zweitveröffentlichung identifiziert durch die Universitätsbibliothek IImenau. “

"Within the academic Open Access Strategy identified for deposition by IImenau University Library."

„Dieser Beitrag ist mit Zustimmung des Rechteinhabers aufgrund einer (DFGgeförderten) Allianz- bzw. Nationallizenz frei zugänglich."

"This publication is with permission of the rights owner freely accessible due to an Alliance licence and a national licence (funded by the DFG, German

Research Foundation) respectively." 


\title{
ECG-Multichannel Frontend for Quick Stimulus Response Based on FPGA with Implemented Real-Time, Online QRS Detection Algorithm
}

\author{
P. Fritzsche ${ }^{1,2}$, S. Niemöller ${ }^{1}$, D. Laqua ${ }^{1}$, P. Husar ${ }^{1}$ \\ ${ }^{1}$ (Department of Biosignal Processing, Ilmenau University of Technology), Ilmenau, Germany \\ 2paul.fritzsche@tu-ilmenau.de
}

\begin{abstract}
Nowadays, economic globalisation is one of the strongest influences in the field of bioelectronics. Due to this, a solid symbiosis is increasing between research and economics. Especially small and medium-sized businesses (SMBs) expect to become and remain a part of this symbiosis. The sector of medical biosignal processing demands effective, compact, low cost and low energy embedded systems that are capable to adapt to new conditions on the market. In this paper, a system solution for SMBs is described that comes with the above-mentioned factors.

For this reason, an easily adaptable multichannel frontend as daughterboard combined with an open source, online, realtime QRS detection algorithm based on Field Programmable Gate Array (FPGA) was developed.
\end{abstract}

\section{Introduction}

The strong influence of economics in the research and the rapid globalization progress has led to a symbiosis between industry and research of similar directions.

The efforts of SMBs are to become and remain a part of this symbiosis. The field of bioelectronics is one of the most important fields that offers possible market entry due to its enormous potential. Medical biosignal processing is a continuously growing area of bioelectronics that demands highest efficiency.

It is crucial for systems in this sector to have the capability of constantly adapting to new conditions on the market. The optimal system should be effective, profitable, compact, energy efficient and also adaptable to new conditions without the loss of any of the abovementioned attributes. The experimental realization is carried out by a highly integrated, embedded system.

FPGAs offer a very good platform for embedded systems. They are suitable for creating monitoring systems or can be very helpful for signal pre-processing for real-time data-monitoring by reducing PC workload. The implementation of algorithms on these embedded systems differ from other computer based monitoring systems. The architecture of an FPGA brings the ability of parallel processing and offers especially for complex algorithms a very high performance. This technology allows the parallel collection of data from ADCs without time multiplexing. Besides that, a great modular variety is given due to hardware programming.

The following system solution for SMBs was developed considering these criteria.

\section{$2 \quad$ Material and Methods}

As shown in figure 1, hardware foundation is an Xilinx Spartan-3AN FPGA. This FPGA is integrated in the NanoBoard 3000 [2] development board designed by Altium. Furthermore, analog, multichannel frontend is provided by several ADS1251 [10] precision, wide dynamic range, delta-sigma, analog-to-digital converters with 24 bit resolution from Texas Instruments.

The real-time, online QRS detection algorithm by Hamilton and Tompkins is implemented into a soft device within the FPGA and the results can be viewed on the on-board TFT. The fundamental for this is the open source ECG analysis project from $\mathrm{P}$. Hamilton [5].

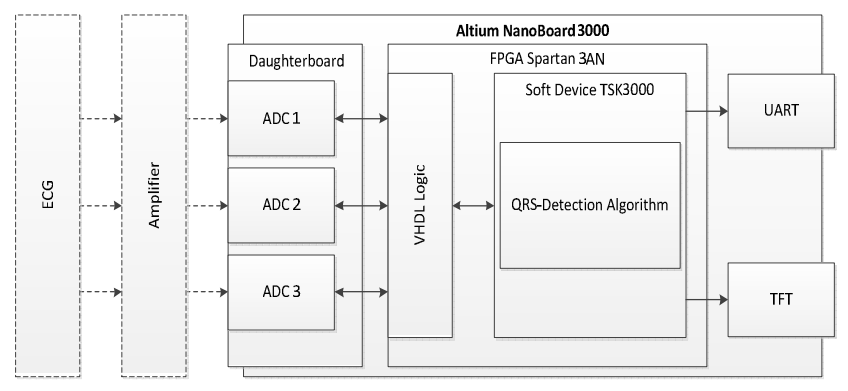

Figure 1, Chart of the system solution containing all the essential hardware and software components. The components within the dashed boxes are not part of the solution.

\subsection{Multichannel Frontend}

The analog, multichannel frontend is provided by several Texas Instruments ADS1251 that are placed on the plugon daughterboard.

The expected dynamic range of the signal, in our case the ECG, has frequency components up to $200 \mathrm{~Hz}$. However, the sampling rate is adjustable in case a later implemented 
algorithm requires oversampling. The amplitude of the incoming ECG signal has to be lifted to $\pm \pm 5 \mathrm{~V}$ range by external amplifiers. Moreover, the interval was chosen to provide optimal range for the option to add an ECG amplifier. In preparation for possible mobile applications an important point is to ensure low energy consumption. This criterion is fulfilled by the ADS1251. The delta-sigma converter with a resolution of 24 bits allows sample rates up to $20 \mathrm{ksps}$ and has a Sync Mode, so that multiple ADCs can be synchronized. After 64 cycles of modulation the mean value is computed. The mean value is available at the Data Out Register (DOR) after every 384 clock cycles. The implemented VHDL logic retrieves the data of all three channels and provides it for the soft device.

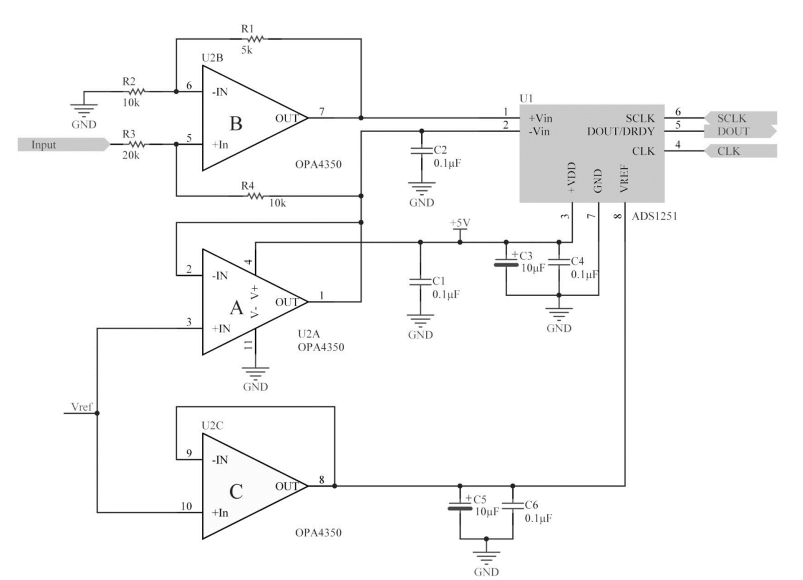

Figure 2, Excerpt block diagram of the plug-on daughterboard. The figure shows the realization of the level shift in combination with the ADS1251 according to Texas Instruments [9].

\subsection{Detection Algorithm}

In 2002, Hamilton published an open source ECG analysis software [5] based on the papers Pan et al. [8] and Hamilton et al. [4]. This is the foundation of the system described here, chosen due to its well-known reliability and performance. The implementation of Hamilton's QRS detection algorithm in the soft device needs an adjustment of the software in order to suit the hardware solution. Therefore some slight modifications of the source code were made.

\subsection{Validation System}

The validation system occupies the major part of any development. Thus, it is of utmost importance to choose a method that is scientifically accepted and comparable.

Since the flexible overall system is specifically designed for the Hamilton et al. QRS detection algorithm, the validation has to ensure the comparability of its results with other QRS detection algorithms.

Orglmeister et al. [6, Table 2] compared the results of various algorithms with regard to the used databases in tabular form.

The choice of a suitable database fell on the MIT-BIH Arrhythmia Database [3] [7]. The reasons are, first, Hamilton
[5, Table 1] tested his algorithm with that database, and secondly, the majority of published algorithms used the MIT-BIH Arrhythmia Database. To provide the system ECGs in its original resolution and in proper range, the PCI card ADLINK6216 [1] was used. The executive program of the data feed and data recording was written in Matlab, as it supports PhysioNet, ADLINK PCI-6216 card and serial ports. The WFDB Toolbox offers the possibility of accessing PhysioNet to download ECGs from the MITBIH Arrhythmia Database. The Toolbox for ADLINK Cards permits its control via Matlab. The written function is responsible for the generation of the signal, receiving the measured data and their storage. The function loads the required ECG signal of the MIT-BIH Arrhythmia Database and transfers it via PCI card ADLINK 6216 to the multichannel frontend. Subsequently after the processing and transfer via the FPGA logic, the implemented algorithm on the soft device handles the further data processing. If required, the data for the measurement protocol is sent over the serial interface RS-232 via UART to a PC. The R-Peak detection can be viewed on the onboard TFT.

The following simplified structure chart (Figure 3) shows the validation chain for a better understanding.

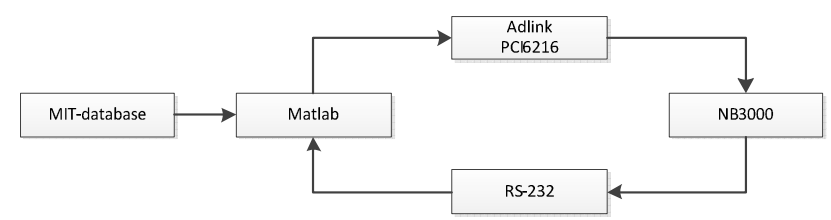

Figure 3, Validation System as block diagram. The figure shows the sequence of the validation system in which the arrows represent the signal path. Matlab is starting and terminal point.

\section{Results}

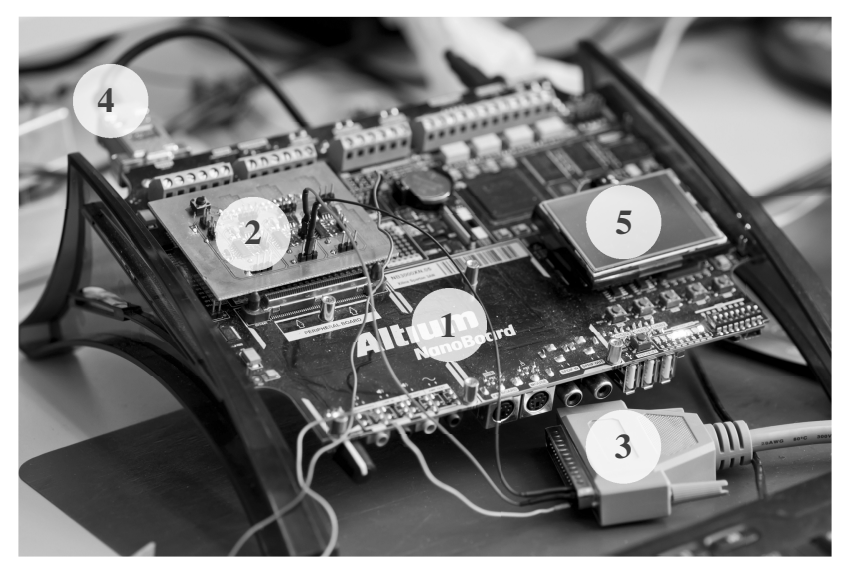

Figure 4, Hardware System. The picture shows the overall system. Foundation is the Altium NanoBoard 3000 (1) with an Xilinx Spartan-3AN FPGA. The developed plugon daughterboard (2) receives the data provided by the PCI ADLINK6216 (3). The processed data is sent over the serial interface (4) while the detection is visualized on the TFT (5) shown in figure 4. 
First the system solution without the algorithm was tested with hybrid data sets and the standard MIT-BIH Arrhythmia Database on channel 2. Figure 5 shows an excerpt of the results of the database file number 100 .

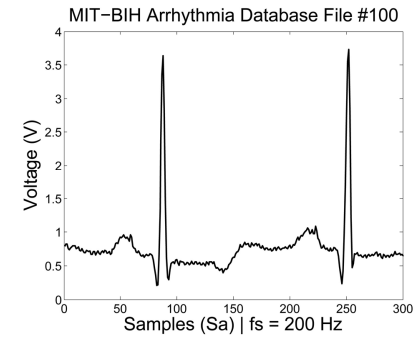

(a)

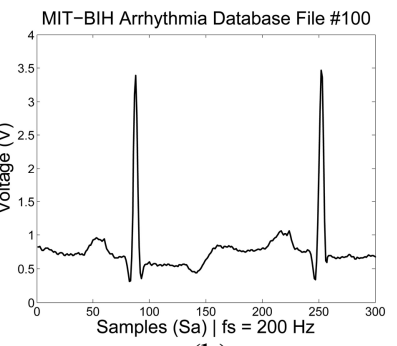

(b)
Figure 5, Results of the system solution without the algorithm. (a) Resampled ECG from the MIT-BIH Arrhythmia Database file number 100. (b) Digitalized ECG from the MIT-BIH Arrhythmia Database file number 100 with removed filter delay of 3 samples of the ADS1251.

Consequently, the total system was then tested with hybrid data sets and the standard MIT-BIH Arrhythmia Database on channel 2. Testing the implemented algorithm in the developed hardware and software solution with the MITBIH Arrhythmia Database led to a sensitivity result of $97.49 \%$ and a positive predictivity of $99.45 \%$. The learning phase and other pathophysiological events were not excluded from the results.

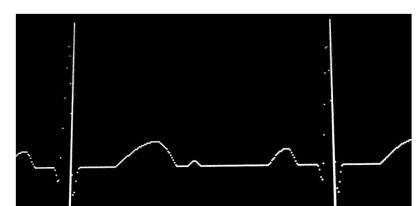

(a)

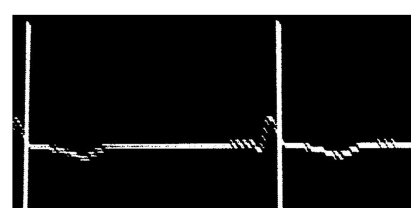

(b)
Figure 6, Picture of the onboard TFT. (a) R-Peak detection of a hybrid dataset. (b) R-Peak detection of a real ECG provided by an external amplifier with a gain of 500 .

\section{Discussion}

The current system setup allows a significant reduction of PC workload for further processing or usage as a fully autonomic monitoring system.

The SMBs have to decide whether to implement the desired system fully in e.g. VHDL and thus losing the capability of fast adaption or to use a soft device and hence gain more flexibility but suffer a loss in parallel processing. However, using a soft device first, saves time and offer the flexibility needed during the development. After a system has been fully optimized and all components are chosen, a full VHDL implementation can follow.

Hamilton achieved a sensitivity of $99.74 \%$ and a positive predictivity of $99.81 \%$ using the original code [5, Table 1]. A better "fine-tuning" of our system and the exclusion of the learning phase will show improvement in the results.

\section{Conclusion}

This paper presents an effective and compact embedded system concept. The results show that a robust stand-alone ECG-multichannel frontend for quick stimulus response based on FPGA with an implemented real-time, online QRS detection algorithm was developed.

The usage of an FPGA not only reduces PC workload and allows individual pre-processing compared to microcontroller systems, it parallel processing is also essential for future embedded hardware systems.

For development reasons, this system is more suitable for $\mathrm{SMBs}$ as it offers lower market entry costs compared to ASICs for odd trading business, because it is highly adaptable due to its plug-on daughterboard and the use of a soft device. The actual QRS detection algorithm is just a first step to a variety of potential algorithms that can be easily implemented in the soft device.

An expansion of the current system with more channels is possible. Furthermore, the development kit can be transferred into a standalone customized biosignal acquisition and real-time processing system.

\section{References}

[1] ADLINK Technology Inc., "Datasheet: PCI/PCIe/cPCI-6208/6216 Series “, URL: http://www.adlinktech.com/PD/marketing/Datas heet/PCI-6208+6216Series/PCI6208+6216Series_Datasheet_en_1.pdf, 2012. Last checked: 30.06.2012. Note: Login required. Altium Limited, "Datasheet: Altium NanoBoard 3000“, URL: http://nb3000.altium.com/PDFs/NB3000\%20Pro duct\%20Data\%20Sheet_en.pdf, 2009.

Last checked: 30.06.2012.

Goldberger, A.L., Amaral, L.A.N. and Glass, L. et al., "PhysioBank, PhysioToolkit, and PhysioNet: Components of a New Research Resource for Complex Physiologic Signals", Circulation, vol. 101, no. 23, pp. e215-e220, 2000.

Hamilton, Patrick S. and Tompkins, Willis J., "Quantitative Investigation of QRS Detection Rules Using the MIT/BIH Arrhythmia Database", IEEE Transactions on Biomedical Engineering, vol. BME-33, no. 12, pp. 1157-1165, 1986.

[5] Hamilton, P., "Open source ECG analysis", Computers in Cardiology, pp. 101-104, 2002.

Kohler, B.-U., Hennig, C. and Orglmeister, R., "The principles of software QRS detection", IEEE Engineering in Medicine and Biology Magazine, vol. 21, no. 1, pp. 42-57, 2002. MIT-BIH Arrhythmia Database", IEEE Engineering in Medicine and Biology Magazine, vol. 20, no. 3, pp. 45-50, 2001. 
[8] Pan, Jiapu and Tompkins, Willis J., “A RealTime QRS Detection Algorithm”, IEEE Transactions on Biomedical Engineering, vol. BME-32, no. 3, pp. 230-236, 1985.

[9] Texas Instruments, "Datasheet: 24-Bit, 20kHz, Low-Power ANALOG-TO-DIGITAL CONVERTER“, URL:

http://www.ti.com/lit/gpn/ads1251, 2009.

Last checked: 30.06 .2012$. 